\title{
A comparative evaluation of gemtuzumab ozogamicin + daunorubicin-cytarabine and other treatments for newly diagnosed acute myeloid leukemia
}

\author{
Ajibade O Ashaye*,1, Zarmina Khankhel ${ }^{1}$, Yingxin Xu1 ${ }^{1}$, Kyle Fahrbach ${ }^{1}$, Ruth Mokgokong ${ }^{2}$, \\ Michelle E Orme ${ }^{3}$, Kathryn Lang ${ }^{2}$, Joseph C Cappelleri ${ }^{4}$ \& Carla Mamolo ${ }^{4}$ \\ ${ }^{1}$ Evidera Inc., Waltham, MA, USA \\ ${ }^{2}$ Pfizer Ltd, Surrey, UK \\ ${ }^{3}$ ICERA Consulting Ltd, Swindon, UK \\ ${ }^{4}$ Pfizer Inc., Groton, CT, USA \\ *Author for correspondence: ajibade_ashaye@yahoo.com
}

\begin{abstract}
Aim: To evaluate the comparative efficacy and safety of gemtuzumab ozogamicin + daunorubicincytarabine $(G O+D A)$ versus common induction therapies for newly diagnosed acute myeloid leukemia. Materials \& methods: A network meta-analysis following a systematic literature review. Results: In basecase analyses, GO + DA was associated with significantly greater overall survival and relapse-free survival versus most comparators, and similar rates of complete remission versus all evaluated comparators. Similar findings were seen in the subgroup analyses. Grade 3+ bleeding and hepatic events were higher with GO + DA versus some comparators, consistent with GO's profile. No differences were found for other evaluated outcomes. Conclusion: GO + DA provides significant overall survival and relapse-free survival benefit versus evaluated induction regimens for newly diagnosed acute myeloid leukemia.
\end{abstract}

Lay abstract: To evaluate the efficacy and safety of gemtuzumab ozogamicin + daunorubicin-cytarabine (GO + DA) compared with common treatments for patients newly diagnosed with acute myeloid leukemia, we systematically reviewed the literature published on the topic, then performed a network meta-analysis. In our base-case analyses, GO + DA was associated with significantly greater overall survival and relapsefree survival versus most comparative treatments, and with similar rates of complete remission versus all evaluated comparators. Grade 3+ bleeding and liver events were higher with GO + DA than with some comparators. These findings are consistent with what is known about the efficacy and safety profile of GO.

First draft submitted: 11 July 2018; Accepted for publication: 11 October 2018; Published online: 19 November 2018

Keywords: acute myeloid leukemia $\bullet$ chemotherapy $\bullet$ gemtuzumab ozogamicin $\bullet$ induction treatment $\bullet$ network meta-analysis $\bullet$ newly diagnosed $\bullet$ oncology $\bullet$ overall survival $\bullet$ relapse-free survival $\bullet$ systematic literature review

Acute myeloid leukemia (AML) is characterized by a malignant proliferation of hematopoietic progenitor cells of the myeloid lineage within the bone marrow. It is the most common form of acute leukemia in adults, with an estimated incidence of about 20,000 new cases in the USA in 2018 [1]. The median age of onset is 67 years, and more than $50 \%$ of cases occur in individuals 65 years of age or older [2].

Treatment of newly diagnosed AML typically includes induction therapy followed by postremission therapy [3]. Induction therapy usually consists of an anthracycline-cytarabine (an antimetabolite) combination, such as daunorubicin-cytarabine (DA), a regimen that has been in use for over 40 years $[4,5]$. This combination is associated with toxicity (including thrombocytopenia, neutropenia and anemia) and marginal rates of complete remission (53-58\%), particularly in older adults [6,7]. Several agents have been evaluated as potential induction therapies, but most did not show any significant benefit compared with DA, the existing standard of care $[8,9]$.

Future Medicine 
Gemtuzumab ozogamicin (GO) is a humanized antibody-drug conjugate composed of a monoclonal antibody targeting CD33, which is covalently linked to a semisynthetic derivative of calicheamicin. It destroys malignant cells by binding to their $\mathrm{CD} 33$ antigen [10]. Once internalized, calicheamicin, the cytotoxic agent, is released to destroy the cell. Originally approved by the US FDA in 2000 for the treatment of relapsed AML, GO was voluntarily withdrawn in 2010 due to safety concerns [11,12]. However, evidence from later studies, such as the AML-15, AML16 and ALFA-0701 trials, showed the administration of a lower, single or fractionated dose of GO $\left(3 \mathrm{mg} / \mathrm{m}^{2}\right)$ in combination with DA was associated with improvements in 2-year estimated event-free survival (EFS), relapse-free survival (RFS) and overall survival (OS) versus DA alone. Additionally, longer-term data confirmed a benefit with GO on EFS and RFS among patients with newly diagnosed de novo or secondary AML. These findings were also documented in a meta-analysis [13]. Based on this new evidence, GO was approved in September 2017 by the FDA as monotherapy or in combination with DA for the treatment of newly diagnosed AML in adults, and for the treatment of relapsed or refractory disease in patients 2 years of age and older [14].

No published trials have compared GO + DA with other induction therapy agents in patients with newly diagnosed de novo or secondary AML. Thus, we conducted a systematic literature review (SLR) and network meta-analysis (NMA) to evaluate the comparative clinical efficacy and safety of GO + DA versus other induction therapies in this patient population.

\section{Materials \& methods}

The SLR was conducted per the guidelines and recommendations of the Cochrane Collaboration [15] and reported according to the Preferred Reporting Items for Systematic Reviews and Meta-Analyses (PRISMA) extension statement for systematic reviews incorporating NMAs [16].

\section{Data sources \& searches}

MEDLINE, Embase, the Cochrane Central Register of Controlled Trials and the Database of Abstracts of Reviews of Effects were searched to identify Phase II or III randomized controlled trials (RCTs) published in English between 1 January 2000 and 29 September 2015. Searches were conducted using a combination of indexing and free-text terms for the population, interventions and study design of interest. In addition, proceedings from oncology conferences, and bibliographies of recent systematic reviews (published between January 2012 and September 2015) were evaluated to identify other relevant publications. The search algorithms for the literature databases are in the online Supplementary Data: Medline in Supplementary Table 1; Embase in Supplementary Table 2; and CENTRAL and DARE in Supplementary Table 3. The list of conferences appears in Supplementary Table 4. An updated search was conducted in January 2018 to identify any Phase II or III RCTs that may have been published in the literature databases since the September 2015 search.

\section{Eligibility criteria}

Full details of the eligibility criteria including interventions and outcomes of interest are provided in Supplementary Table 5. RCTs that evaluated adult subjects with newly diagnosed de novo or secondary AML receiving induction therapy were included in the review. RCTs were excluded if they focused on pediatric subjects, or on subjects with relapsed or refractory AML, newly diagnosed AML not receiving induction therapy (e.g., induction plus maintenance, or maintenance treatment only), myelodysplastic syndrome (MDS) and/or refractory anemia with excess blasts (RAEB), or acute promyelocytic leukemia. In addition, studies that did not investigate any of the interventions of interest or were not RCTs were excluded. Outcomes of interest included survival end points (e.g., OS, RFS), remission (e.g., complete remission, complete remission with incomplete platelet recovery) and safety or tolerability outcomes. Outcomes as defined by each of the included studies appear in Supplementary Table 6.

\section{Study selection, data extraction \& risk-of-bias assessment}

The titles and abstracts of the citations identified from the searches were evaluated using the prespecified criteria. Records that met the inclusion criteria following the title and abstract screening were further evaluated based on the content of their full texts. Screening was conducted independently by two investigators at each stage; disagreements were resolved by discussion with a third investigator. Data on study, patient and treatment characteristics and outcomes of interest were extracted by one investigator and validated for accuracy by a second investigator. Risk of 
bias was also assessed independently by two investigators using the Cochrane Collaboration's Risk of Bias Assessment Tool [17].

\section{Data synthesis \& analysis}

An assessment was conducted to evaluate the feasibility of conducting a comparative analysis using an NMA. This included a comprehensive evaluation of the study and patient characteristics, potential effect modifiers (e.g., cytogenetics), consistency in the definition and availability of outcomes, and any clinical heterogeneity. The assessment also sought to determine if the assumptions required for NMAs, such as homogeneity (equivalence of treatment effects across trials within each pairwise comparison), transitivity (the validity of making indirect comparisons) and consistency (the equivalence of direct and indirect evidence) were met.

Estimates of relative effects were derived via fixed-effect (FE) and random-effects (RE) models in a Bayesian framework, following established techniques for integrating hazard ratios (HRs) and count data via an NMA [18-23]. The FE model results were considered the base-case given the limited data available to estimate the betweenstudy variation of treatment effects that are required for a RE model. Where RE analyses were feasible, a vaguely informative prior $(\mathrm{U}[0,1])$ on this $\mathrm{RE}$ variance component was used. Base-case analyses included all studies providing evidence for a specific outcome of interest. Two subgroup analyses were also conducted: an analysis limited to studies evaluating a 100\% de novo AML population; and an analysis limited to studies evaluating GO alone or in combination with DA or any other therapy to provide a GO versus no-GO comparison.

The analyses included only outcomes for which there were sufficient data, with little or no heterogeneity in study and patient characteristics, and a connected evidence network. Evidence networks for the base-case analyses are provided in Supplementary Figures 1 and 2.

The following outcomes were included in the base-case and subgroup analyses: OS, RFS, complete remission, overall response, induction death, relapse, resistant disease and safety outcomes (grade 3+ infection, skin effects, hepatic events and bleeding events). Subgroup analyses were not feasible for overall response, induction death or safety outcomes. Except for RFS and OS, all outcomes were analyzed using the number of patients with at least one event as the numerator, while the denominator was dependent on the individual study's methods. HRs were extracted for time-to-event end points (RFS and OS) when reported and log-transformed for the analyses. When HRs were available only in Kaplan-Meier curves, these were digitized and data points were extracted using established methods [24,25].

For each NMA, a burn-in of 50,000 samples was produced to ensure convergence was achieved before producing a further 50,000 samples for parameter estimation [26]. The median of the posterior distribution was reported as the point estimate (HR or odds ratio [OR]), and the corresponding $95 \%$ credible intervals (CrIs) were obtained with the 2.5th and 97.5th percentiles of the posterior distribution.

Global treatment rankings were estimated based on the Surface Under the Cumulative Ranking Curve (SUCRA) values [23]. SUCRA values reflect the percentage of area under the plot of likelihood of cumulative rankings (1st, 1 st or 2 nd, 1 st or 2 nd or 3rd, etc., depending on the number of treatments examined). They enable the global ranking of treatments overall for an outcome by calculating the HR/OR for each drug compared with GO + DA, and counting the proportion of iterations in which each drug had the most favorable HR/OR compared with GO + DA. The SUCRA would be $100 \%$ when a treatment is certain to be the best of those observed and $0 \%$ when a treatment is certain to be the worst. Thus, when discussing treatment rankings, we are comparing treatments on total SUCRA area as opposed to any specific probability of being the best, or second-best, or third-best treatment, etc.

Analyses were conducted using OpenBUGS v3.2.3, which has coding identical to WinBUGS, and Rv3.3.0 [26]. Model convergence was confirmed via inspection of the ratios of Monte Carlo error to the standard deviation of the simulations (an approximation of the standard deviation of the posterior distribution) and evaluation of the three-chain Brooks-Gelman-Rubin plots [27,28]. Model fit was explored by comparing the deviance information criterion (DIC) and the posterior mean of the residual deviance for the FE model and RE model [29].

\section{Results}

\section{Study characteristics}

In total, 1398 unique records were identified from the database searches. Sixty RCTs were reviewed, 17 of which (reported in 24 publications) provided data for the NMA. A PRISMA flow diagram detailing the screening process is shown in Figure 1. Key trial characteristics are presented in Table 1. Most of the trials were Phase III, multicenter 


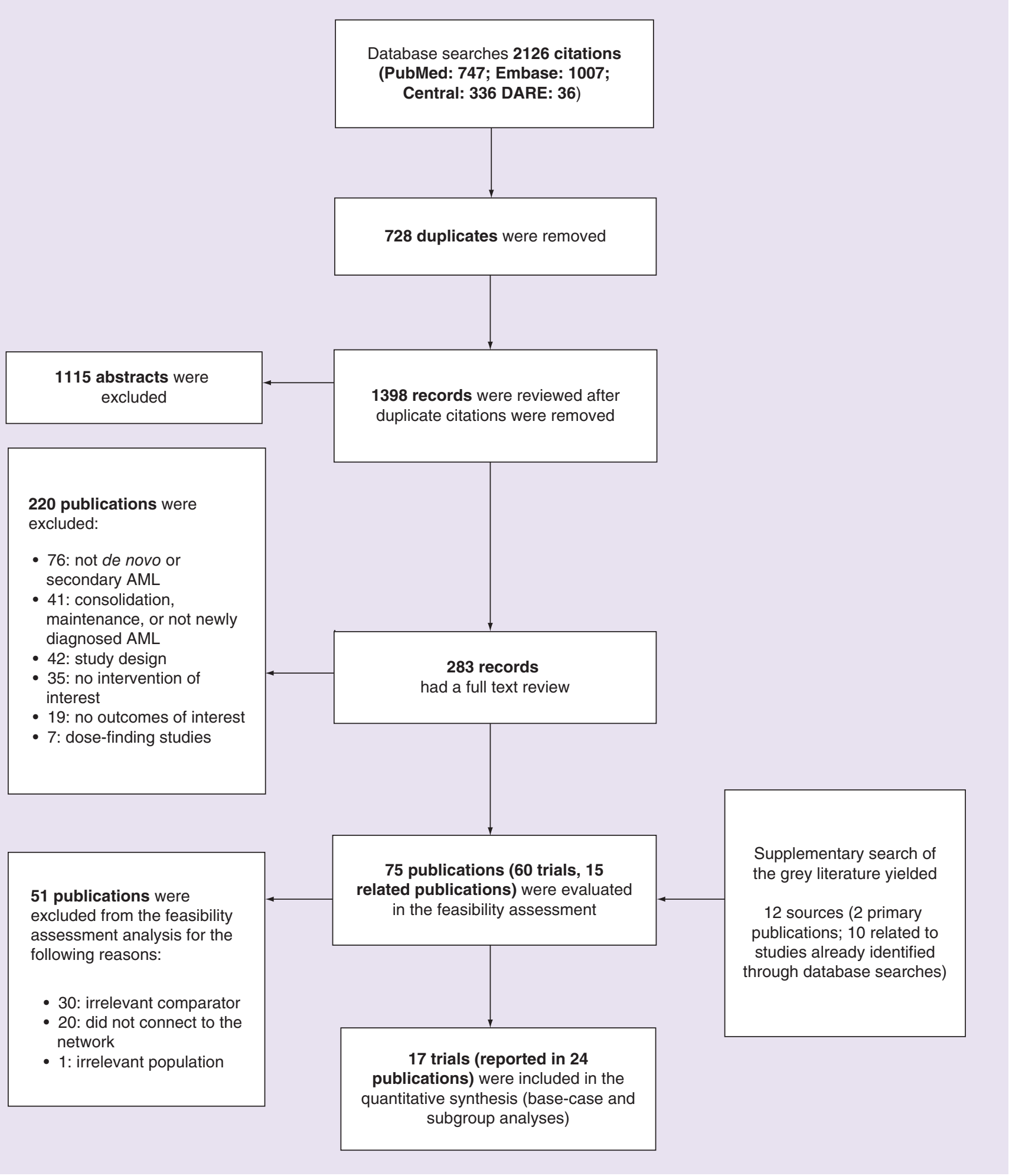

Figure 1. Preferred Reporting Items for Systematic Reviews and Meta-Analyses flow chart. 


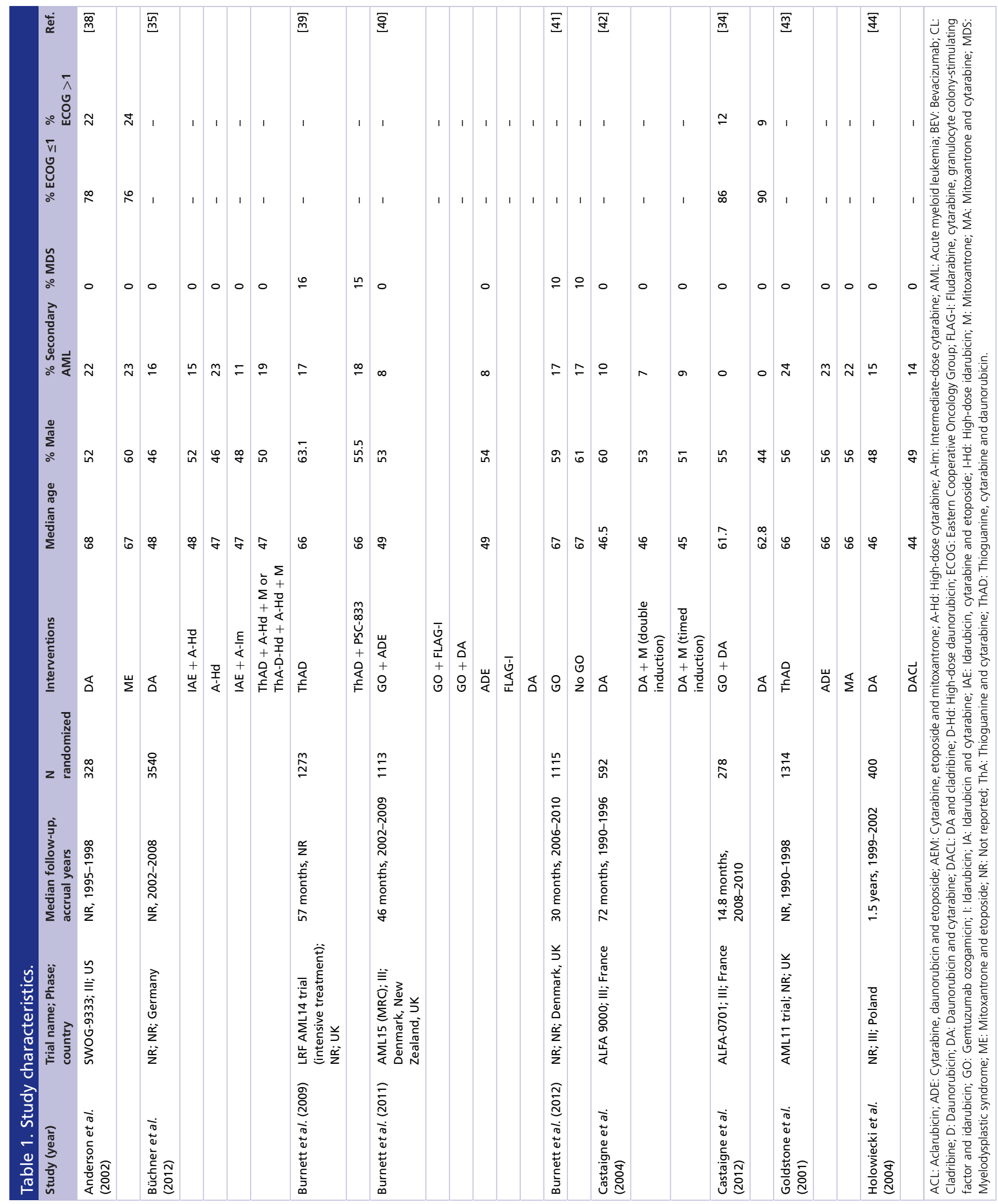




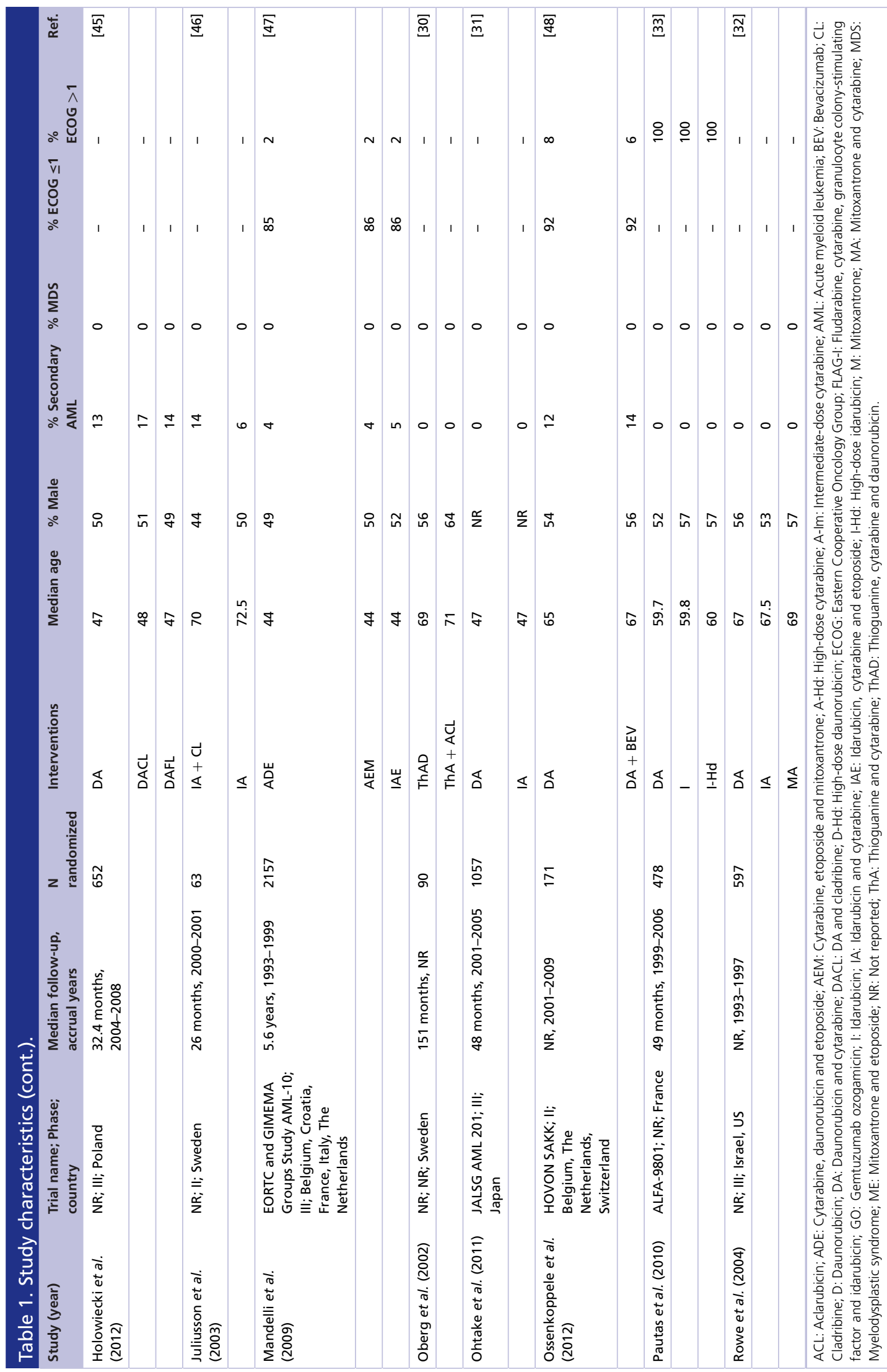


studies. The number of patients randomized across the 17 trials ranged from 63 to 3540 , for a total of sample size of 15,218 . The median patient ages at baseline were between 44 and 72.5 years, and the proportion of males ranged from 44 to 64\%. Median study follow-up was between 14.8 and 151 months, when such data were reported.

Most patients evaluated in the trials had de novo AML (73-100\%), with five trials enrolling patients with de novo AML exclusively [30-34]. Of the studies that included secondary AML, the range was from 4 to $24 \%$ of the study population. Mutation status was sparsely reported; only two studies detailed the proportion of patients with the presence of nucleophosmin mutations (32-64\% of patients were positive) and FLT3 mutations (16-36\% of patients were positive) $[34,35]$. Studies were found to be sufficiently similar for gender and prognostic factors (e.g., the proportion of patients with de novo or secondary AML, MDS or RAEB; performance criteria/status and cytogenetic criteria).

\section{Risk of bias}

A detailed summary of the risk of bias assessment is presented in Supplementary Table 7. It was difficult to assess some studies due to insufficient reporting of information and inaccessibility of study protocols or clinical study reports. Eight of the 17 trials were judged to have used an adequate method of randomization; there was limited information about randomization methods in the remaining nine trials. Fourteen of 17 studies were judged to be at unclear risk of bias for allocation concealment: studies seldom reported information on blinding of study participants, caregivers or outcomes assessors. Three studies were deemed to have a high risk of bias because they used open-label designs. Most trials (82\%) used an intention-to-treat approach for analyzing survival outcomes.

\section{Efficacy outcomes}

OS \& induction death

OS was significantly higher with GO + DA compared with DA alone, idarubicin + cytarabine (IA) or mitoxantrone + etoposide (ME; Table 2). No treatment significantly outperformed GO + DA with respect to OS. The probability for GO + DA to be better than comparators in the base-case OS analyses ranged from $49.8 \%$ (vs $\mathrm{DA}+$ cladribine [DACL]) to $99.9 \%$ (vs ME; Figure 2). Results of the subgroup analyses were consistent with the base-case analyses, showing that GO-based regimens were significantly superior to comparators with respect to OS in the $100 \%$ de novo subgroup analysis (Table 3), as well as in the GO versus no-GO analysis (Table 4).

With regard to induction death, the probability for GO + DA to be better than comparators ranged from $2 \%$ (vs idarubicin) to $67.5 \%$ (vs high-dose cytarabine [A-Hd]) in the base-case analyses (Table $2 \&$ Figure 3). Subgroup analyses were not feasible for the induction death outcome. Point estimates from the RE model were consistent with that of the FE model for both outcomes (results of RE model not shown).

\section{Relapse-free survival}

In the base-case analyses, RFS was significantly higher among patients who received GO + DA compared with all other evaluated treatments, including the following: DA; A-Hd; DACL; DA + fludarabine (DAFL); IA; idarubicin + cytarabine + cladribine (IACL); idarubicin + cytarabine + etoposide (IAE) + A-Hd; mitoxantrone + cytarabine (MA); ME; and thioguanine + cytarabine + daunorubicin (ThAD) + A-Hd + mitoxantrone (M) (Table 2). The probability for GO + DA to be better than comparators in the base-case analyses ranged from $99.5 \%$ (vs DAFL) to $100 \%$ (vs DA and vs IAE + A-Hd; Figure 4). Similar findings were seen in the $100 \%$ de novo AML (Table 4) and GO vs no-GO subgroup analyses (Table 5). Point estimates from the FE and RE models were consistent (results of RE model not shown).

\section{Complete remission}

Rates of complete remission were similar between GO + DA and the evaluated comparators in both the base-case (Table 2) and subgroup analyses (Tables $3 \& 4$ ). The probability for GO + DA to be better than comparators ranged from $5.5 \%$ (vs idarubicin) to $91.1 \%$ (vs ME) in the base-case analyses (Figure 5) and from 5.5 to $60.5 \%$ in both subgroup analyses (Tables $3 \& 4$ ).

\section{Overall response}

Overall response was similar between GO + DA and the evaluated comparators in the base-case analyses. The probability for GO + DA to be better than comparators ranged from 22.1 to $90.8 \%$ (Table $2 \&$ Figure 6). Subgroup analyses were not feasible for the overall response outcome. 
Table 2. Network meta-analysis results: base-case - efficacy outcomes and induction death (fixed-effects).

\begin{tabular}{|c|c|c|c|c|c|c|c|}
\hline Comparisons & $\begin{array}{l}\text { OS } \\
\mathrm{HR}, 95 \% \mathrm{Crl}\end{array}$ & $\begin{array}{l}\text { RFS } \\
\text { HR, 95\% Crl }\end{array}$ & $\begin{array}{l}\text { Induction death } \\
\text { OR, } 95 \% \mathrm{Crl}\end{array}$ & $\begin{array}{l}\text { Complete } \\
\text { remission } \\
\text { OR, } 95 \% \mathrm{Crl}\end{array}$ & $\begin{array}{l}\text { Overall response } \\
\text { OR, } 95 \% \mathrm{Crl}\end{array}$ & $\begin{array}{l}\text { Relapse } \\
\text { OR, } 95 \% \text { Crl }\end{array}$ & $\begin{array}{l}\text { Resistant disease } \\
\text { OR, } 95 \% \mathrm{Crl}\end{array}$ \\
\hline $\mathrm{GO}+\mathrm{DA}$ vs DA & $0.69(0.49-0.97)^{\dagger}$ & $0.52(0.36-0.75)^{\dagger}$ & $1.89(0.64-6.31)$ & $1.08(0.63-1.83)$ & $1.47(0.83-2.62)$ & $0.77(0.57-1.04)$ & $0.52(0.27-1.01)$ \\
\hline $\mathrm{GO}+\mathrm{DA}$ vs $\mathrm{ADE}$ & - & - & $1.33(0.34-5.58)$ & $1.09(0.50-2.41)$ & - & $0.73(0.43-1.24)$ & - \\
\hline $\mathrm{GO}+\mathrm{DA}$ vs A-Hd & $0.70(0.46-1.05)$ & $0.53(0.34-0.83)^{\dagger}$ & $0.74(0.21-2.84)$ & - & $1.20(0.62-2.36)$ & - & $1.07(0.49-2.31)$ \\
\hline $\mathrm{GO}+\mathrm{DA}$ vs AlE & - & - & $1.27(0.28-5.95)$ & $1.18(0.53-2.69)$ & - & - & - \\
\hline $\mathrm{GO}+\mathrm{DA}$ vs AME & - & - & $1.46(0.32-6.90)$ & $1.03(0.46-2.37)$ & - & - & - \\
\hline GO + DA vs BEV & - & - & - & $1.04(0.45-2.38)$ & - & - & - \\
\hline $\begin{array}{l}\mathrm{GO}+\mathrm{DA} \text { vs } \\
\mathrm{DA}+\mathrm{M}^{\ddagger}\end{array}$ & - & - & $1.34(0.39-5.05)$ & $1.07(0.53-2.18)$ & - & $0.85(0.49-1.47)$ & $0.85(0.33-2.22)$ \\
\hline $\begin{array}{l}\mathrm{GO}+\mathrm{DA} v \mathrm{~s} \\
\mathrm{DA}+\mathrm{M}^{\S}\end{array}$ & - & - & $1.29(0.37-4.85)$ & $1.24(0.61-2.51)$ & - & $1.14(0.65-1.98)$ & $0.65(0.25-1.65)$ \\
\hline $\mathrm{GO}+\mathrm{DA}$ vs DACL & $1.00(0.62-1.61)$ & $0.61(0.39-0.95)^{\dagger}$ & $1.75(0.54-6.19) \mathbb{I}$ & $0.77(0.42-1.40)$ & - & - & $0.99(0.45-2.18)$ \\
\hline GO + DA vs DAFL & $0.71(0.45-1.13)$ & $0.51(0.31-0.85)^{\dagger}$ & $2.30(0.66-8.64) \mathbb{I}$ & $1.01(0.53-1.93)$ & - & - & $0.56(0.26-1.22)$ \\
\hline GO + DA vs FLAG-I & - & - & - & - & - & $1.29(0.89-1.87)$ & - \\
\hline $\begin{array}{l}\mathrm{GO}+\mathrm{DA} v \mathrm{~s} \\
\mathrm{GO}+\mathrm{ADE}\end{array}$ & - & - & - & - & - & $0.69(0.39-1.20)$ & - \\
\hline $\begin{array}{l}\text { GO + DA vs } \\
\text { GO + FLAG-I }\end{array}$ & - & - & - & - & - & $1.53(1.05-2.24)^{\dagger}$ & - \\
\hline GO + DA vs I & - & - & $4.99(1.07-26.47) \mathbb{I}$ & $0.54(0.25-1.15)$ & - & - & $0.89(0.36-2.16)$ \\
\hline GO + DA vs IA & $0.68(0.47-0.99)^{\dagger}$ & $0.54(0.36-0.80)^{\dagger}$ & $1.31(0.36-5.13)$ & $0.97(0.46-2.02)$ & - & - & - \\
\hline $\mathrm{GO}+\mathrm{DA}$ vs IACL & - & - & $0.99(0.08-9.11) \mathbb{I}$ & $0.87(0.23-3.28)$ & - & - & - \\
\hline $\begin{array}{l}\mathrm{GO}+\mathrm{DA} v s \\
\mathrm{IAE}+\mathrm{A}-\mathrm{Hd}\end{array}$ & $0.71(0.48-1.05)$ & $0.42(0.27-0.65)^{\dagger}$ & $1.57(0.45-5.96)$ & - & $1.14(0.60-2.19)$ & - & $0.70(0.33-1.45)$ \\
\hline $\begin{array}{l}\mathrm{GO}+\mathrm{DA} v \mathrm{~s} \\
\mathrm{IAE}+\mathrm{A}-\mathrm{Im}\end{array}$ & $0.74(0.48-1.15)$ & $0.51(0.32-0.81)^{\dagger}$ & $0.90(0.25-3.60)$ & - & $0.75(0.37-1.54)$ & - & $1.79(0.75-4.25)$ \\
\hline $\mathrm{GO}+\mathrm{DA}$ vs I-Hd & - & - & $2.63(0.63-11.94) \mathbb{I}$ & $0.74(0.35-1.54)$ & - & - & $0.73(0.31-1.77)$ \\
\hline $\mathrm{GO}+\mathrm{DA}$ vs MA & $0.77(0.50-1.18)$ & $0.46(0.26-0.80)^{\dagger}$ & $2.27(0.60-9.23) \mathbb{I}$ & $0.88(0.42-1.85)$ & - & - & - \\
\hline $\mathrm{GO}+\mathrm{DA}$ vs $\mathrm{ME}$ & $0.52(0.34-0.80)^{\dagger}$ & $0.43(0.24-0.75)^{\dagger}$ & $1.97(0.49-8.52) \mathbb{I}$ & $1.61(0.81-3.22)$ & - & $0.48(0.18-1.23)$ & $0.36(0.16-0.79)^{\dagger}$ \\
\hline GO + DA vs ThAD & - & - & $2.49(0.62-10.51)^{\mathbb{I}}$ & $0.66(0.30-1.46)$ & - & - & - \\
\hline $\begin{array}{l}\text { GO + DA vs } \\
T h A+A C L\end{array}$ & - & - & - & $080(0.25-2.52)$ & - & - & - \\
\hline $\begin{array}{l}\text { GO + DA vs } \\
\text { ThAD + A-Hd + M }\end{array}$ & $0.69(0.46-1.03)$ & & $0.80(0.23-3.02)$ & - & $1.08(0.57-2.09)$ & - & $1.18(0.55-2.48)$ \\
\hline \multicolumn{8}{|c|}{$\begin{array}{l}\text { †Values represent statistically significant results based on the } 95 \% \text { Crl. } \\
\text { ¥Double induction. } \\
\text { §Timed induction. } \\
\text { \#A value less than } 1 \text { signifies an advantage for GO + DA. } \\
\text { IIImprecisely estimated comparisons. } \\
\text { ACL: Aclarubicin; ADE: Cytarabine, daunorubicin and etoposide; AEM: Cytarabine, etoposide and mitoxantrone; A-Hd: High-dose cytarabine; A-Im: Intermediate-dose cytarabine; } \\
\text { BEV: Bevacizumab; Crl: Credible interval; DA: Daunorubicin and cytarabine; DACL: Daunorubicin, cytarabine and cladribine; DAFL: Daunorubicin, cytarabine and fludarabine; FE: } \\
\text { Fixed-effect; GO: Gemtuzumab ozogamicin; HR: Hazard ratio; I: Idarubicin; IA: Idarubicin and cytarabine; IACL: Idarubicin, cytarabine and cladribine; IAE: Idarubicin, cytarabine and } \\
\text { etoposide; I-Hd: High-dose idarubicin; M: Mitoxantrone; MA: Mitoxantrone and cytarabine; ME: Mitoxantrone and etoposide; OR: Odds ratio; OS: Overall survival; RFS: Relapse-free } \\
\text { survival; ThA: Thioguanine and cytarabine; ThAD: Thioguanine, cytarabine and daunorubicin. }\end{array}$} \\
\hline
\end{tabular}

Relapse \& resistant disease

Patients treated with GO + DA generally had similar rates of relapse and resistant disease versus those receiving comparator treatments, with two exceptions (Table 2). First, a significantly higher relapse rate (OR: 1.53; 95\% CrI [1.05-2.24]) was found for GO + DA versus the treatment GO + FLAG-I (fludarabine, cytarabine, granulocyte colony-stimulating factor and idarubicin). The second exception was when GO + DA significantly outperformed $\mathrm{GO}+\mathrm{ME}$ with regard to resistant disease (OR: 0.36; 95\% CrI [0.16-0.79]; Figures $7 \&$ 8). In the base-case analyses, the probability that GO + DA would be better than comparators ranged from 1.3\% (vs GO + FLAG-I) to $95.8 \%$ (vs DA) for relapse, and from $9.2 \%$ (vs IAE + A-Im) to $99.4 \%$ (vs ME) for resistant disease (Figures $7 \&$ 8); that same probability ranged from 60.3 to $97.5 \%$ for resistant disease in the $100 \%$ de novo analyses (Table 3 ). 


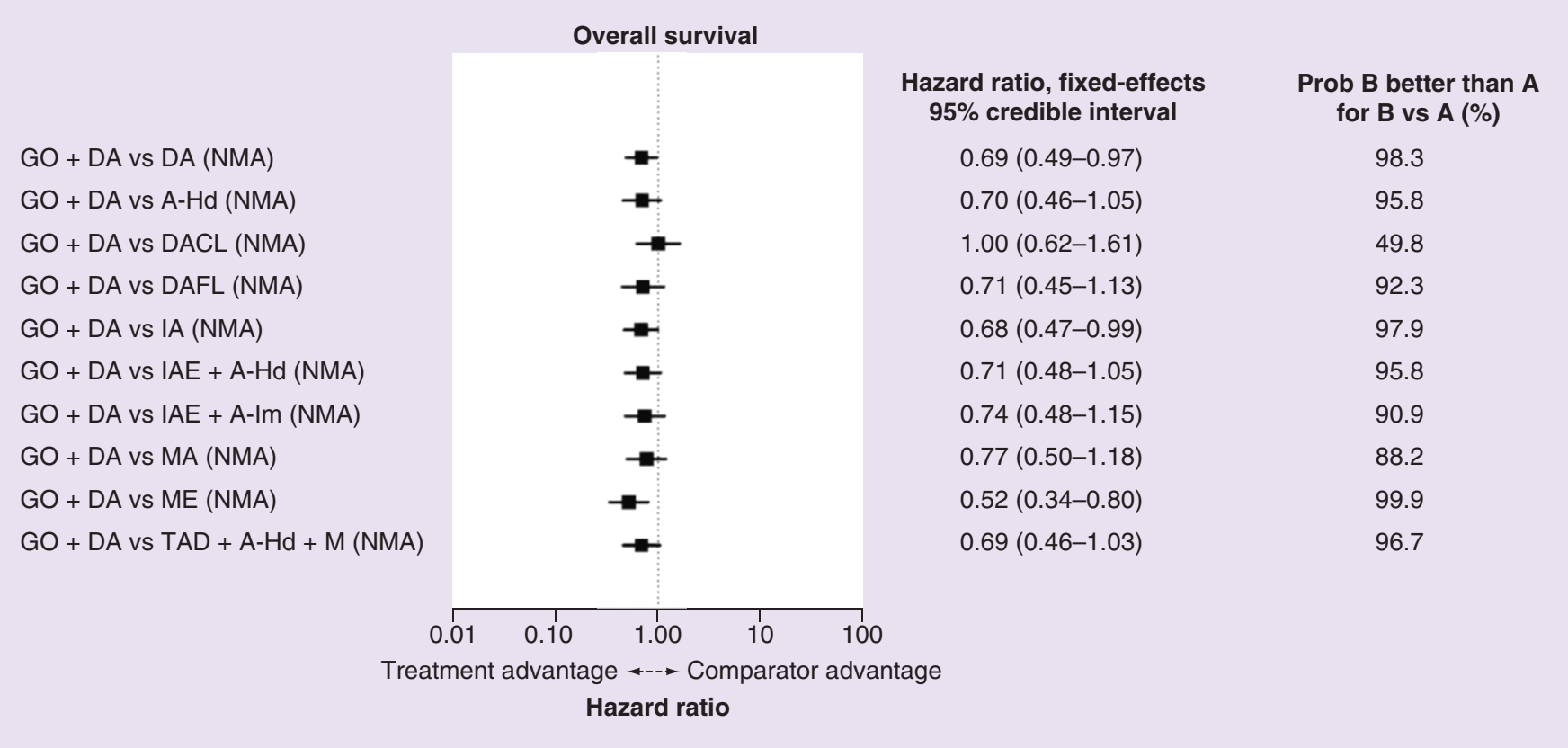

Figure 2. Base-case analysis: overall survival.

${ }^{\dagger}$ A value less than 1 signifies an advantage for GO + DA.

ADE: Cytarabine, daunorubicin and etoposide; AME: Cytarabine, etoposide and mitoxantrone; A-Hd: High-dose cytarabine; A-Im: Intermediate-dose cytarabine; DA: Daunorubicin and cytarabine; DACL: Daunorubicin, cytarabine and cladribine; DAFL: Daunorubicin, cytarabine and fludarabine; GO: Gemtuzumab ozogamicin; I: Idarubicin; IA: Idarubicin and cytarabine; IACL: Idarubicin, cytarabine and cladribine; IAE: Idarubicin, cytarabine and etoposide; I-Hd: High-dose idarubicin; M: Mitoxantrone; MA: Mitoxantrone and cytarabine; ME: Mitoxantrone and etoposide; NMA: Network meta-analysis; ThAD: Thioguanine, cytarabine and daunorubicin.

\section{Table 3. Network meta-analysis results: $100 \%$ de novo analyses (fixed-effects).}

\begin{tabular}{|c|c|c|c|c|}
\hline Comparisons & $\begin{array}{l}\text { OS } \\
\text { HR, } 95 \% \text { Crls }\end{array}$ & $\begin{array}{l}\text { RFS } \\
\text { HR, } 95 \% \text { Crls }\end{array}$ & $\begin{array}{l}\text { Complete remission } \\
\text { OR, } 95 \% \text { Crls }\end{array}$ & $\begin{array}{l}\text { Resistant disease } \\
\text { OR, 95\% Crls }\end{array}$ \\
\hline $\mathrm{GO}+\mathrm{DA}$ vs DA & $\begin{array}{l}0.69(0.49-0.97)^{\dagger} \\
98.2 \%\end{array}$ & $\begin{array}{l}0.52(0.36-0.75)^{\dagger} \\
100 \%\end{array}$ & $\begin{array}{l}1.07(0.63-1.83) \\
60.5 \%\end{array}$ & $\begin{array}{l}0.53(0.27-1.00) \\
97.5 \%\end{array}$ \\
\hline GO + DA vs I & - & - & $\begin{array}{l}0.54(0.25-1.15) \\
5.4 \%\end{array}$ & $\begin{array}{l}0.89(0.37-2.16) \\
60.3 \%\end{array}$ \\
\hline$G O+D A$ vs IA & $\begin{array}{l}0.68(0.47-0.99)^{\dagger} \\
97.8 \%\end{array}$ & $\begin{array}{l}0.54(0.36-0.80)^{\dagger} \\
99.9 \%\end{array}$ & $\begin{array}{l}0.96(0.46-2.02) \\
45.6 \%\end{array}$ & - \\
\hline $\mathrm{GO}+\mathrm{DA}$ vs I-Hd & - & - & $\begin{array}{l}0.73(0.35-1.53) \\
20.5 \%\end{array}$ & $\begin{array}{l}0.74(0.31-1.76) \\
75.4 \%\end{array}$ \\
\hline $\mathrm{GO}+\mathrm{DA}$ vs MA & $\begin{array}{l}0.77(0.50-1.18)^{\dagger} \\
88.5 \%\end{array}$ & $\begin{array}{l}0.45(0.26-0.80)^{\dagger} \\
99.7 \%\end{array}$ & $\begin{array}{l}0.87(0.41-1.83) \\
35.7 \%\end{array}$ & - \\
\hline
\end{tabular}

Values in table represent point estimate, credible intervals and probability for treatment (GO + DA) being better than comparator.

$\dagger$ Values represent statistically significant results based on the $95 \% \mathrm{Crl}$.

Crl: Credible interval; DA: Daunorubicin and cytarabine; GO: Gemtuzumab ozogamicin; HR: Hazard ratio; I: Idarubicin; IA: Idarubicin and cytarabine; I-Hd: High-dose idarubicin; MA: Mitoxantrone and cytarabine; OR: Odds ratio; OS: Overall survival; RFS: Relapse-free survival.

\section{Table 4. Network meta-analysis results: gemtuzumab ozogamicin versus no-gemtuzumab ozogamicin analyses}

\section{(fixed-effects)}

\begin{tabular}{|c|c|c|c|c|c|}
\hline Comparison & $\begin{array}{l}\text { OS } \\
\text { HR, } 95 \% \mathrm{Crl}\end{array}$ & $\begin{array}{l}\text { RFS } \\
\text { HR, 95\% CrI }\end{array}$ & $\begin{array}{l}\text { CR } \\
\text { OR, } 95 \% \text { Crl }\end{array}$ & $\begin{array}{l}\text { Relapse } \\
\text { OR, 95\% CrI }\end{array}$ & $\begin{array}{l}\text { Resistant disease } \\
\text { OR, } 95 \% \mathrm{Crl}\end{array}$ \\
\hline Go vs no-GO & $\begin{array}{l}0.87(0.79-0.96)^{\dagger} \\
99.6 \%\end{array}$ & $\begin{array}{l}0.82(0.73-0.91)^{\dagger} \\
100 \%\end{array}$ & $\begin{array}{l}0.97(0.74-1.27) \\
41.3 \%\end{array}$ & $\begin{array}{l}0.82(0.65-1.03) \\
96 \%\end{array}$ & $\begin{array}{l}0.81(0.64-1.03) \\
96 \%\end{array}$ \\
\hline
\end{tabular}

Values in table represent point estimate, credible intervals and probability for treatment (GO) being better than comparator (no-GO).

$\dagger$ Values represent statistically significant results based on the $95 \% \mathrm{Crl}$.

CR: Complete remission; Crl: Credible interval; GO: Gemtuzumab ozogamicin; HR: Hazard ratio; OR: Odds ratio; OS: Overall survival; RFS: Relapse-free survival. 


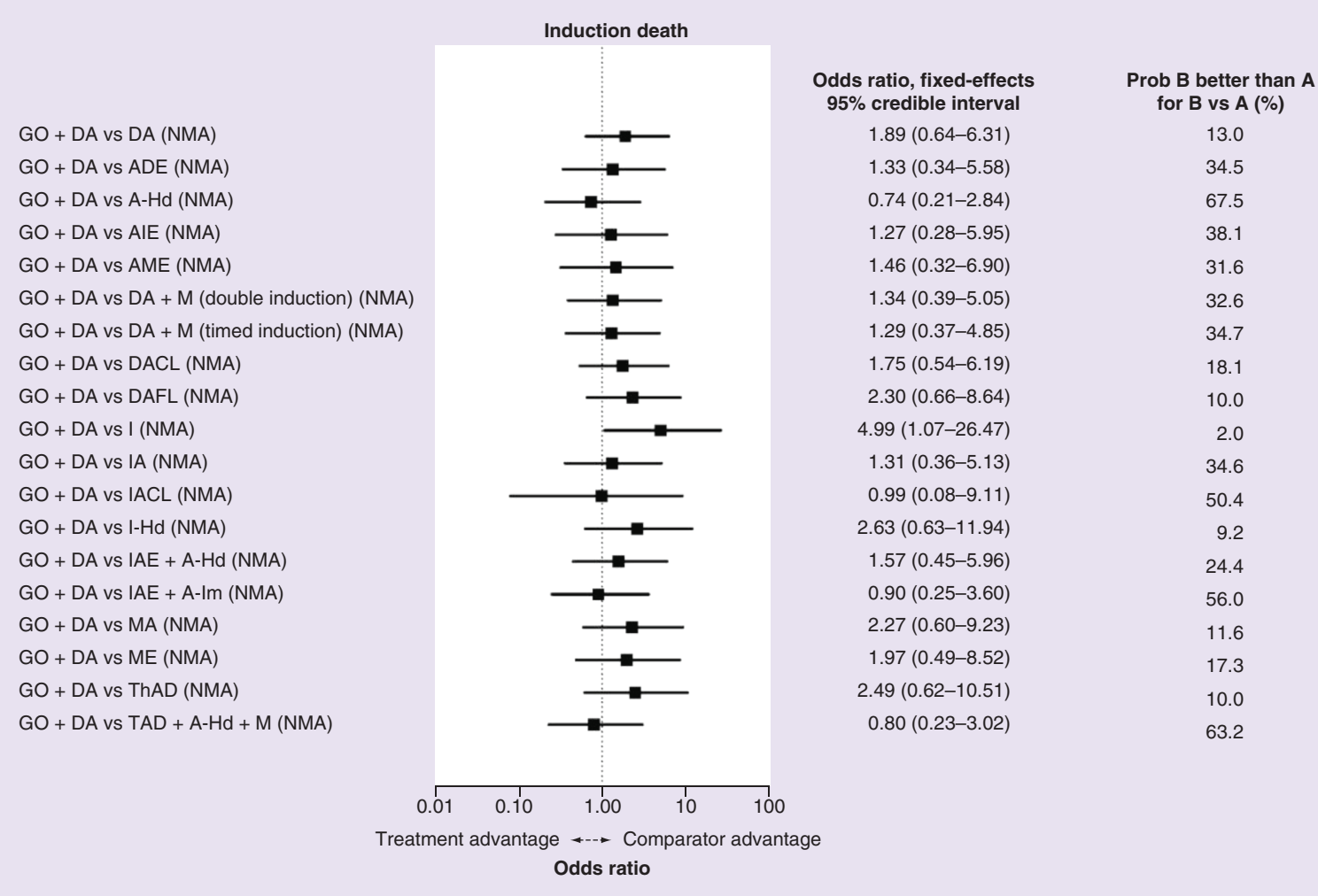

Figure 3. Base-case analysis: induction death.

${ }^{\dagger} \mathrm{A}$ value less than 1 signifies an advantage for $\mathrm{GO}+\mathrm{DA}$.

ADE: Cytarabine, daunorubicin and etoposide; A-Hd: High-dose cytarabine; A-Im: Intermediate-dose cytarabine; AME: Cytarabine, etoposide and mitoxantrone; DA: Daunorubicin and cytarabine; DACL: Daunorubicin, cytarabine and cladribine; DAFL: Daunorubicin, cytarabine and fludarabine; GO: Gemtuzumab ozogamicin; I: Idarubicin; IA: Idarubicin and cytarabine; IACL: Idarubicin, cytarabine and cladribine; IAE: Idarubicin, cytarabine and etoposide; I-Hd: High-dose idarubicin; M: Mitoxantrone; MA: Mitoxantrone and cytarabine; ME: Mitoxantrone and etoposide; NMA: Network meta-analysis; ThAD: Thioguanine, cytarabine and daunorubicin.

\begin{tabular}{|c|c|c|c|c|}
\hline Comparison & $\begin{array}{l}\text { Infection } \\
\text { OR, } 95 \% \mathrm{Crl}\end{array}$ & $\begin{array}{l}\text { Bleeding events } \\
\text { OR, } 95 \% \mathrm{Crl}\end{array}$ & $\begin{array}{l}\text { Skin effects } \\
\text { OR, } 95 \% \mathrm{Crl}\end{array}$ & $\begin{array}{l}\text { Hepatic events } \\
\text { OR, } 95 \% \mathrm{Crl}\end{array}$ \\
\hline GO + DA vs DA & $\begin{array}{l}1.31(0.81-2.15) \\
13.3 \%\end{array}$ & $\begin{array}{l}3.33(1.10-12.33)^{\dagger} \mathbb{I} \\
1.7 \%\end{array}$ & $\begin{array}{l}1.37(0.77-2.49) \\
14.6 \%\end{array}$ & $\begin{array}{l}2.18(0.96-5.35) \\
3.2 \%\end{array}$ \\
\hline $\mathrm{GO}+\mathrm{DA}$ vs $\mathrm{DA}+\mathrm{M}^{\ddagger}$ & $\begin{array}{l}1.06(0.54-2.10) \\
42.9 \%\end{array}$ & - & - & - \\
\hline $\mathrm{GO}+\mathrm{DA}$ vs $\mathrm{DA}+\mathrm{M}^{\S}$ & $\begin{array}{l}1.20(0.62-2.38) \\
29.2 \%\end{array}$ & - & - & - \\
\hline GO + DA vs BEV & - & $\begin{array}{l}7.93(1.99-36.49)^{\dagger} \mathbb{I} \\
0.1 \%\end{array}$ & - & - \\
\hline $\mathrm{GO}+\mathrm{DA}$ vs $\mathrm{DACL}$ & $\begin{array}{l}1.35(0.77-2.37) \\
14.9 \%\end{array}$ & $\begin{array}{l}3.24(0.87-14.20)^{\mathbb{I}} \\
4.1 \%\end{array}$ & $\begin{array}{l}1.77(0.84-3.82) \\
6.8 \%\end{array}$ & $\begin{array}{l}1.87(0.71-5.13) \\
10.7 \%\end{array}$ \\
\hline GO + DA vs DAFL & $\begin{array}{l}1.18(0.65-2.17) \\
29.0 \%\end{array}$ & $\begin{array}{l}4.23(0.94-21.52) \mathbb{I} \\
3.1 \%\end{array}$ & $\begin{array}{l}1.18(0.55-2.59) \\
33.4 \%\end{array}$ & $\begin{array}{l}3.30(1.14-10.19)^{\dagger} \mathbb{I} \\
1.4 \%\end{array}$ \\
\hline GO + DA vs IA & - & $\begin{array}{l}4.18(1.17-17.87)^{\dagger} \mathbb{I} \\
1.4 \%\end{array}$ & - & - \\
\hline \multicolumn{5}{|c|}{$\begin{array}{l}\text { †Values represent statistically significant results based on the } 95 \% \mathrm{Crl} \text {. } \\
\text { ‡Double induction. } \\
\text { §Timed induction. } \\
\mathbb{I} \text { Imprecisely estimated comparisons. } \\
\text { A value less than } 1 \text { signifies an advantage for GO + DA. } \\
\text { BEV: Bevacizumab; Crl: Credible interval; DA: Daunorubicin and cytarabine; DACL: Daunorubicin, cytarabine and cladribine; DAFL: Daunorubicin, cytarabine and fludarabine; GO: Gem- } \\
\text { tuzumab ozogamicin; IA: Idarubicin and cytarabine; M: Mitoxantrone; OR: Odds ratio. }\end{array}$} \\
\hline
\end{tabular}




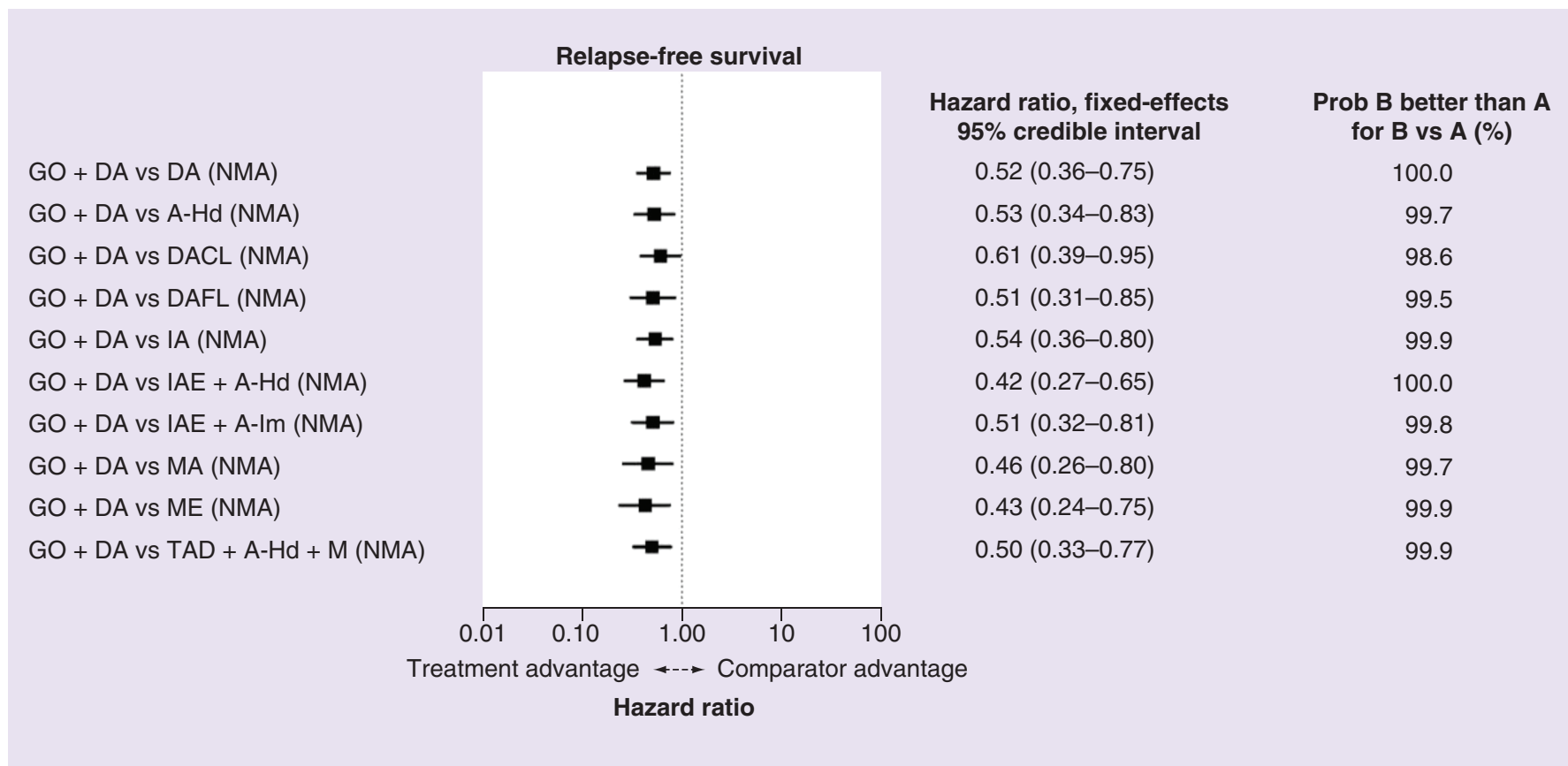

Figure 4. Base-case analysis: relapse-free survival.

${ }^{\dagger} \mathrm{A}$ value less than 1 signifies an advantage for GO + DA.

A-Hd: High-dose cytarabine; A-Im: Intermediate-dose cytarabine; DA: Daunorubicin and cytarabine; DACL: Daunorubicin, cytarabine and cladribine; DAFL: Daunorubicin, cytarabine and fludarabine; GO: Gemtuzumab ozogamicin; IA: Idarubicin and cytarabine; IAE: Idarubicin, cytarabine and etoposide; M: Mitoxantrone; MA: Mitoxantrone and cytarabine; ME: Mitoxantrone and etoposide; NMA: Network meta-analysis; ThAD: Thioguanine, cytarabine and daunorubicin.

Rates of relapse and resistant disease were similar between GO + DA and comparators in both subgroup analyses (Tables $3 \& 4$ ); relapse was not evaluable in the $100 \%$ de novo subgroup analysis.

\section{Safety outcomes}

Patients receiving GO + DA versus comparator treatments had relatively similar odds of experiencing grade 3+ infections and skin effects (Table 5). Rates of bleeding events were significantly higher with GO + DA than with DA alone (OR: 3.33; 95\% CrI [1.10-12.33]), bevacizumab (BEV; 7.93 [1.99-36.49]) or IA (4.18 [1.17-17.87]). Additionally, patients on GO + DA were significantly more likely to experience a hepatic event versus those receiving DAFL. The point estimates were consistent with those of the RE analysis. Safety outcomes were not evaluable for either subgroup analysis.

\section{Discussion}

The purpose of this research was to conduct a comprehensive SLR followed by an NMA to evaluate the comparative efficacy and safety of GO + DA and other agents used as induction therapy for newly diagnosed de novo or secondary AML.

Findings from our analyses revealed that GO-based regimens were similar to comparators with a few key exceptions. In the base-case analyses, GO + DA led to significantly greater OS (six studies; $\mathrm{N}=7218$ ) and RFS (five studies; $\mathrm{N}=6626$ ) than the evaluated comparators. GO + DA was one of the highest ranked treatments for OS (SUCRA 90.5\%), RFS (SUCRA 99.7\%) and overall response (SUCRA 61.5\%) in the evidence network for the base-case analyses (Table 6). Of note, although treatment with GO + DA led to greater OS compared with DA, IA or ME, there was no significant difference with respect to rates of induction death. Considering that OS favors GO + DA, the induction death findings in our analyses may not represent the true survival treatment effect of $\mathrm{GO}+\mathrm{DA}$, due to the small number of deaths occurring during induction therapy. GO + DA was similar to evaluated treatments with regard to relapse rate; however, it was significantly outperformed by GO + FLAG-I (four studies; $\mathrm{N}=2461$ ). 


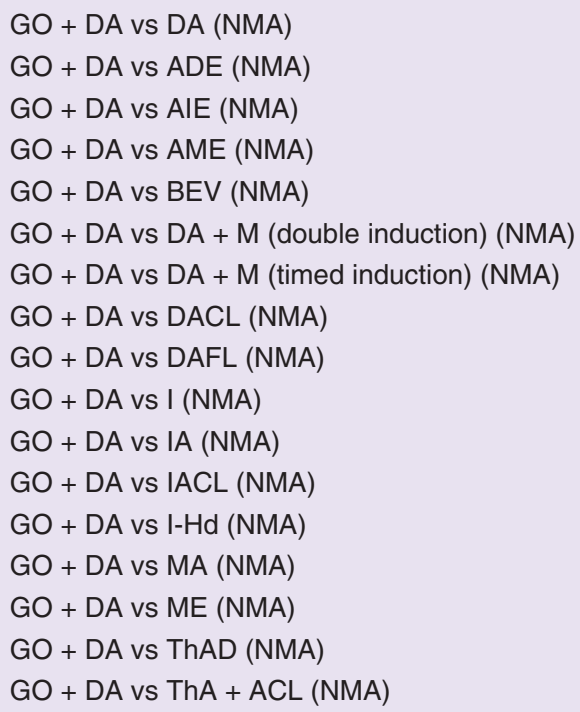

\section{Complete remission}

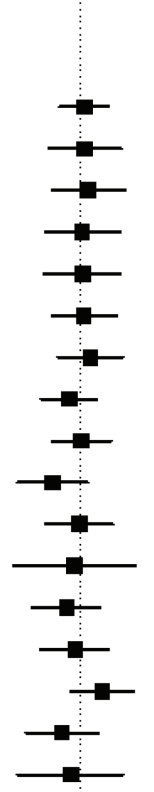

Odds ratio, fixed-effects $95 \%$ credible interval

$1.08(0.63-1.83)$

$1.09(0.50-2.41)$

$1.18(0.53-2.69)$

$1.03(0.46-2.37)$

$1.04(0.45-2.38)$

$1.07(0.53-2.18)$

$1.24(0.61-2.51)$

$0.77(0.42-1.40)$

$1.01(0.53-1.93)$

$0.54(0.25-1.15)$

$0.97(0.46-2.02)$

$0.87(0.23-3.28)$

$0.74(0.35-1.54)$

$0.88(0.42-1.85)$

$1.61(0.81-3.22)$

$0.66(0.30-1.46)$

$0.80(0.25-2.52)$
Prob B better than A for $B$ vs $A$ (\%)

61.0

58.7

65.8

53.2

53.8

57.4

72.4

19.5

51.4

5.5

46.4

41.9

20.7

36.8

91.1

15.5

35.0

\section{$0.01 \quad 0.10 \quad 1.00 \quad 10 \quad 100$}

Comparator advantage $\rightarrow$ Treatment advantage

\section{Odds ratio}

Figure 5. Base-case analysis: complete remission.

${ }^{\dagger}$ A value greater than 1 signifies an advantage for GO + DA.

A-Hd: High-dose cytarabine; A-Im: Intermediate-dose cytarabine; DA: Daunorubicin and cytarabine; DACL: Daunorubicin, cytarabine and cladribine; DAFL: Daunorubicin, cytarabine and fludarabine; GO: Gemtuzumab ozogamicin; I: Idarubicin; IA: Idarubicin and cytarabine; IAE: Idarubicin, cytarabine and etoposide; M: Mitoxantrone; MA: Mitoxantrone and cytarabine; ME: Mitoxantrone and etoposide; NMA: Network meta-analysis; ThAD: Thioguanine, cytarabine and daunorubicin.

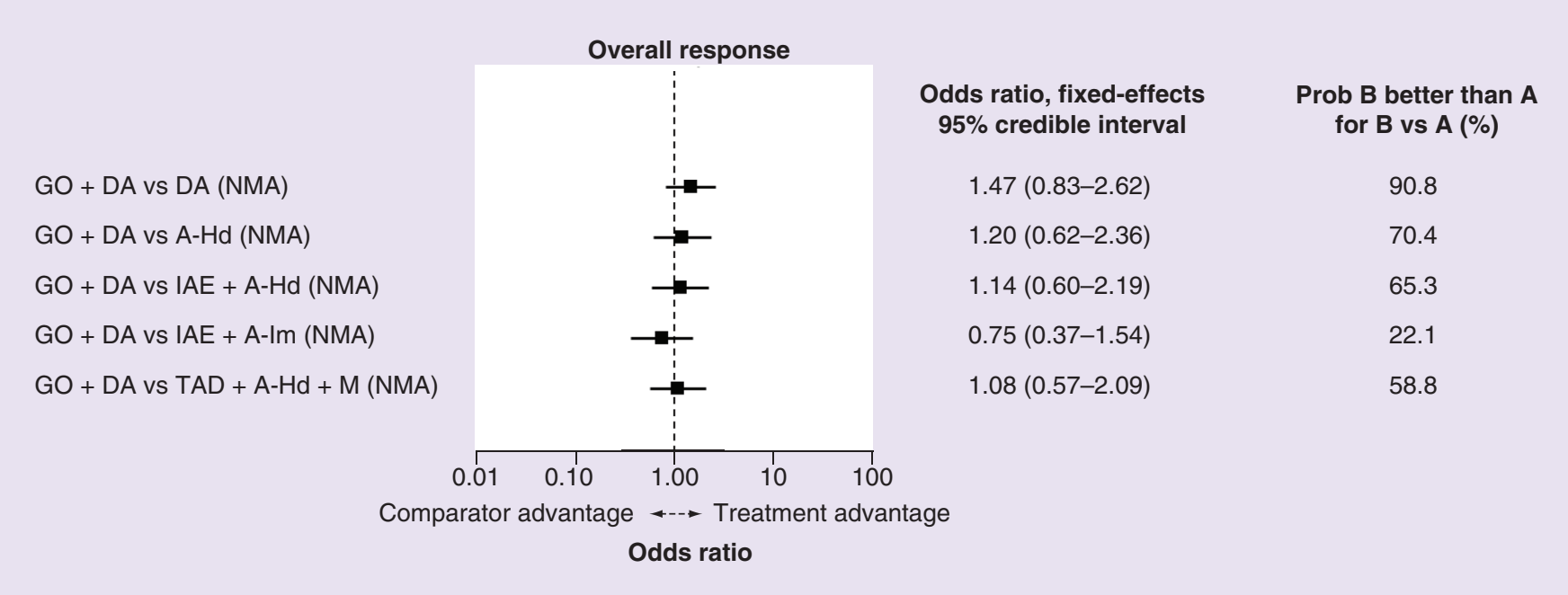

Figure 6. Base-case analysis: overall response.

${ }^{\dagger}$ A value greater than 1 signifies an advantage for GO + DA.

A-Hd: High-dose cytarabine; A-Im: Intermediate-dose cytarabine; DA: Daunorubicin and cytarabine; GO: Gemtuzumab ozogamicin; IAE: Idarubicin, cytarabine and etoposide; M: Mitoxantrone. 
$\mathrm{GO}+\mathrm{DA}$ vs DA (NMA)

$\mathrm{GO}+\mathrm{DA}$ vs ADE (NMA)

$\mathrm{GO}+\mathrm{DA}$ vs DA + M (double induction) (NMA)

$\mathrm{GO}+\mathrm{DA}$ vs DA + M (timed induction) (NMA)

GO + DA vs FLAG-I (NMA)

$\mathrm{GO}+\mathrm{DA}$ vs ME (NMA)

$\mathrm{GO}+\mathrm{DA}$ vs GO + ADE (NMA)

$\mathrm{GO}+\mathrm{DA}$ vs GO + FLAG-I (NMA)
Relapse

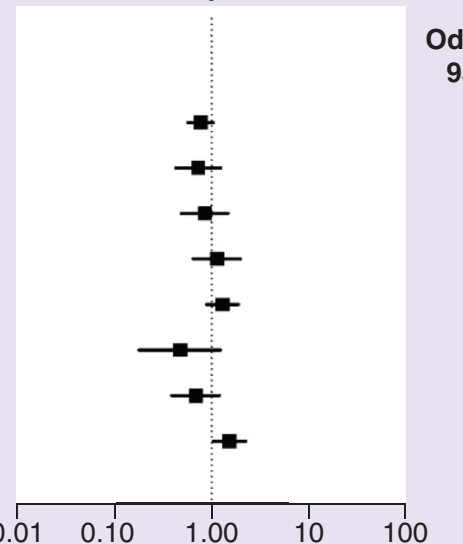

Treatment advantage $\rightarrow$ Comparator advantage
Odds ratio, fixed-effects $95 \%$ credible interval

$0.77(0.57-1.04)$

$0.73(0.43-1.24)$

$0.85(0.49-1.47)$

$1.14(0.65-1.98)$

$1.29(0.89-1.87)$

$0.48(0.18-1.23)$

$0.69(0.39-1.20)$

$1.53(1.05-2.24)$
Prob B better than A

for $B$ vs $A$ (\%)

95.8

88.1

72.4

32.5

9.3

93.6

90.8

1.3

\section{Odds ratio}

Figure 7. Base-case analysis: relapse.

${ }^{\dagger}$ A value less than 1 signifies an advantage for GO + DA.

ADE: Cytarabine, daunorubicin and etoposide; DA: Daunorubicin and cytarabine; FLAG-I: Fludarabine, cytarabine, granulocyte colony-stimulating factor and idarubicin; GO: Gemtuzumab ozogamicin; M: Mitoxantrone; ME: Mitoxantrone and etoposide; NMA: Network meta-analysis.

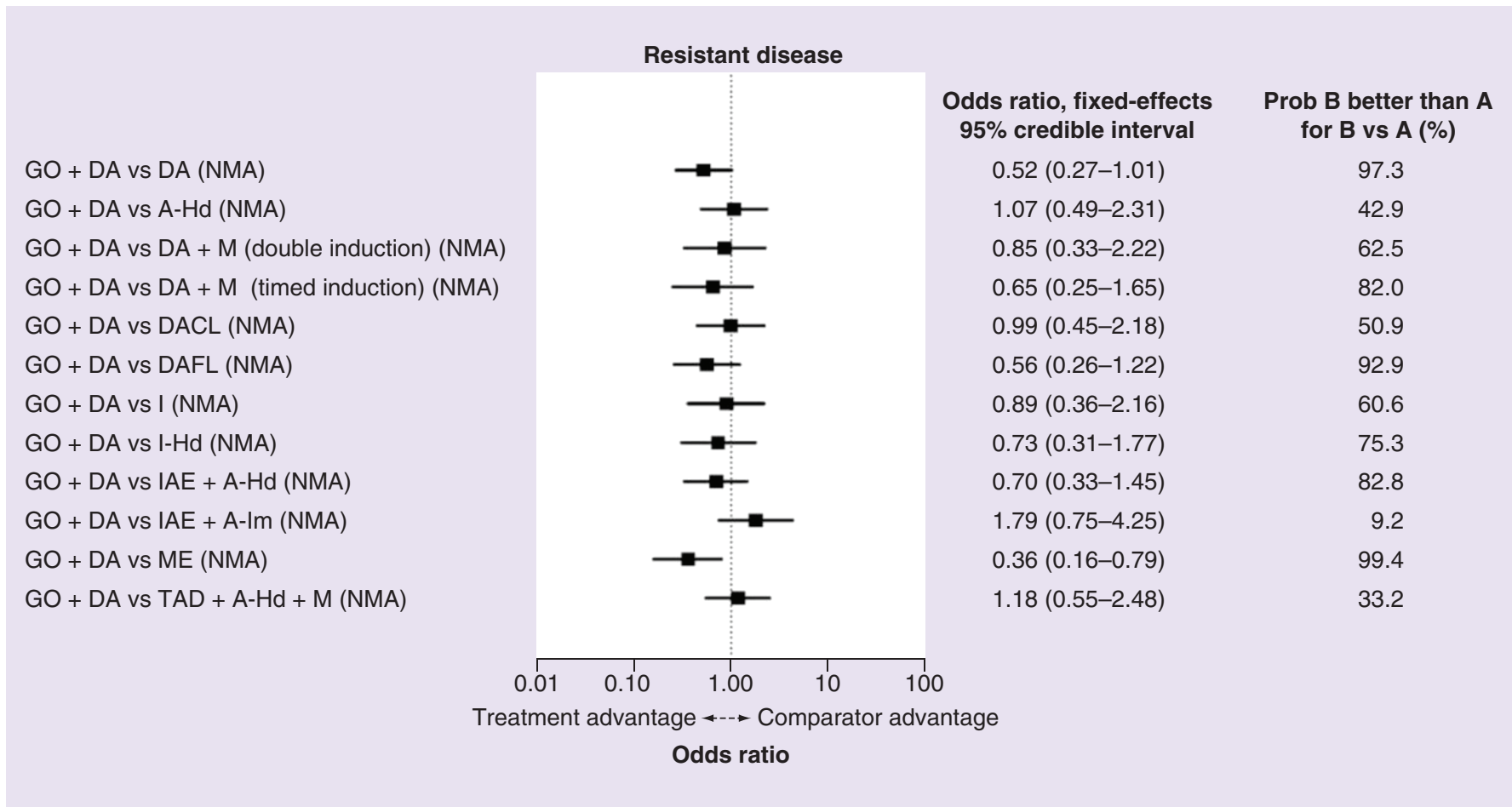

Figure 8. Base-case analysis: resistant disease.

${ }^{\dagger}$ A value less than 1 signifies an advantage for GO + DA.

A-Hd: High-dose cytarabine; A-Im: Intermediate-dose cytarabine; DA: Daunorubicin and cytarabine; DACL: Daunorubicin, cytarabine and cladribine; DAFL: Daunorubicin, cytarabine and fludarabine; FLAG-I: Fludarabine, cytarabine, granulocyte colony-stimulating factor and idarubicin; IAE: Idarubicin, cytarabine and etoposide; I-Hd: High-dose idarubicin; GO: Gemtuzumab ozogamicin; M: Mitoxantrone; ME: Mitoxantrone and etoposide; NMA: Network meta-analysis; ThAD: Thioguanine, cytarabine and daunorubicin. 


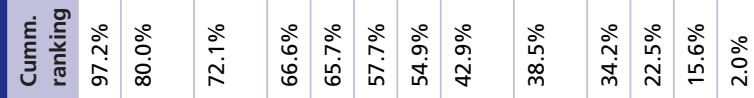

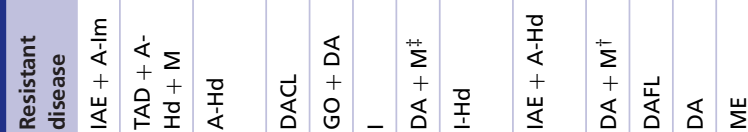

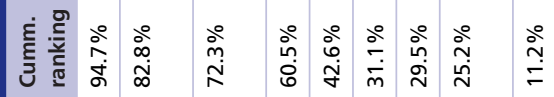

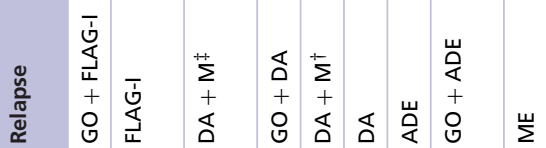

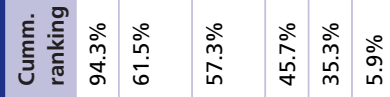

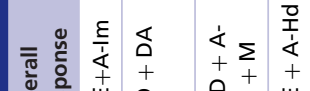

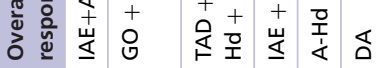

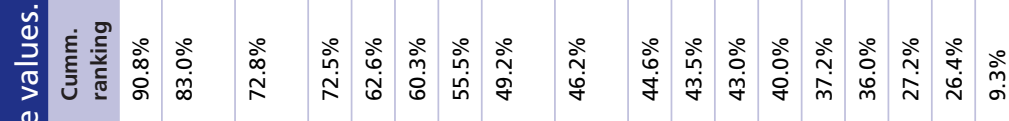

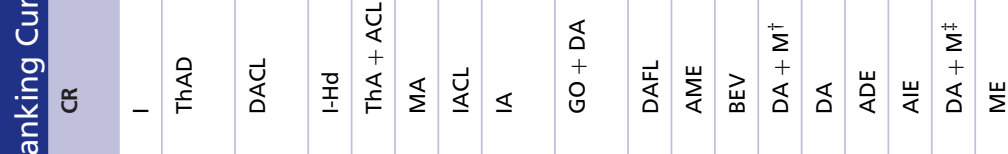

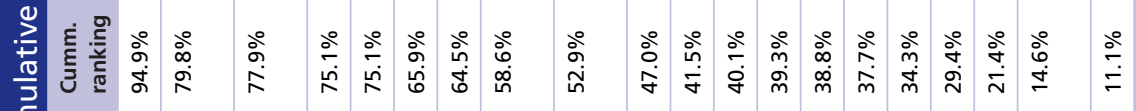

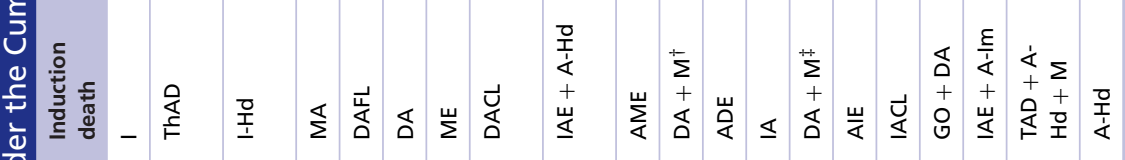

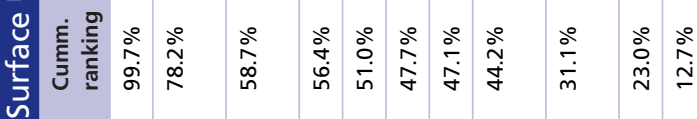

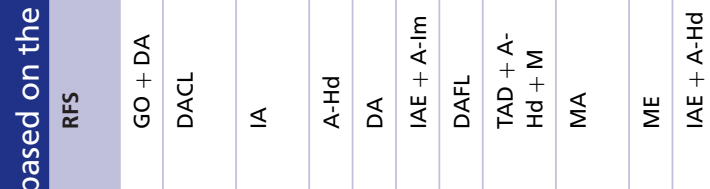

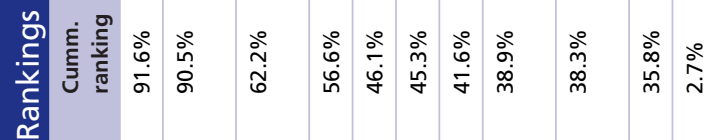

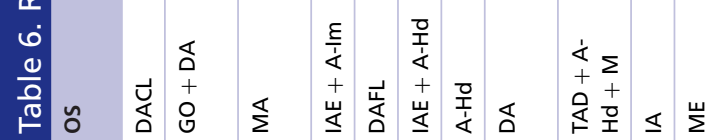

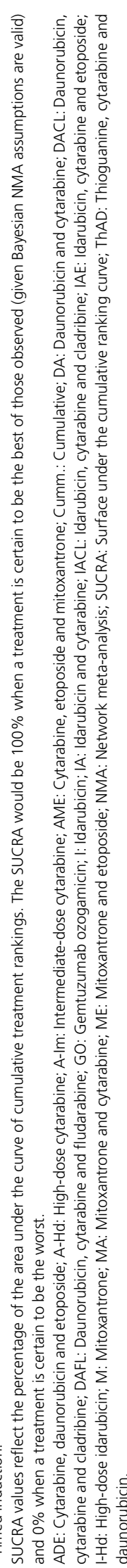


GO + DA was one of the highly ranked treatments for relapse (SUCRA 60.5\%), and resistant disease (SUCRA $65.7 \%$ ) in the evidence network for the base-case analyses (Table 6). Results of the safety analyses showed patients on GO + DA were significantly more likely to experience a bleeding event (five studies; $\mathrm{N}=2558$ ) compared with DA, BEV or IA; a hepatic event (three studies; $N=1330$ ) compared with DAFL; and had a similar frequency of infections and skin effects compared with all other treatments evaluated. This is consistent with the known safety profile of GO.

The $100 \%$ de novo subgroup analyses and the GO versus no-GO subgroup analyses showed that GO + DA and GO-based regimens were significantly more likely to lead to greater OS and RFS benefits compared with other treatments evaluated in the NMA. Results of these analyses were mostly consistent with the base-case. Subgroup analyses were not feasible for overall response, induction death or for the safety outcomes.

Our analyses included data from GO studies that randomized patients to $3 \mathrm{mg} / \mathrm{m}^{2}$ of GO, regardless of the dosing schedule (two studies used a single dose, while a single study used a fractionated dose). Previously, one 'GO versus no-GO' meta-analysis by Hills et al. also compared GO plus chemotherapy with chemotherapy alone, among patients with de novo or secondary AML [13]. However, unlike our analyses, the meta-analysis by Hills et al. differentiated studies evaluating single or fractionated $3 \mathrm{mg} / \mathrm{m}^{2}$ dosing schedules and, within the meta-analysis, provided a combined effect estimate for studies evaluating the single $3 \mathrm{mg} / \mathrm{m}^{2}$ dose of GO. Despite this difference, results between the two analyses were similar and found that the addition of GO to various chemotherapy regimens led to OS and RFS benefits. Some of the findings of the Hills et al.'s study corroborated those in an earlier meta-analysis by Kharfan-Dabaja et al., which evaluated the benefits and harms of adding GO to conventional chemotherapy for induction treatment of AML [36]. Like Hills et al.'s meta-analysis, Kharfan-Dabaja et al. reported that the addition of GO to conventional chemotherapy improved RFS and EFS. However, in their analyses, GO combination therapy did not impact OS, but significantly increased early mortality [36].

Our review and analyses have various strengths. To our knowledge, this is the first study evaluating the comparative efficacy and safety of GO + DA versus other induction therapies based on an NMA. Previous studies, such as Kharfan-Dabaja et al. and Hills et al. were based on traditional pairwise meta-analyses limited to GO-based trials $[13,36]$. In our study, estimates for relative efficacy and safety were derived from a Bayesian analytical framework, following an SLR conducted with the rigor recommended by established guidelines. This ensures that all relevant comparators have been identified and included in the analyses.

Additionally, the Bayesian approach allows for the estimation of treatment rankings for outcomes of interest and based on values from the SUCRA, which takes the uncertainty in treatment effects into account. We also explored the effects of using RE as well FE models for the analyses to further evaluate the validity of our NMA results and better assess the robustness of our findings. DICs were typically similar between the FE and RE models, as would be expected for sparse networks with little apparent heterogeneity and low power to detect heterogeneity. The FE models had marginally better DICs (lower DIC values) than the RE model; results (estimates and CrIs) were often extremely similar between both models.

There are also some limitations to our analyses. Not all studies provided data for every effect modifier or prognostic variable, such as patients' performance status; the proportion of patients with secondary AML, MDS or RAEB; cytogenetic risk status; or the presence of nucleophosmin and FLT3 mutations. Hence, effect modification based on these factors cannot be ruled out. Similarly, the efficacy of supportive care has changed over time, and could impact relative effects; unfortunately, the fact that most comparisons were investigated in only one study precluded investigation of this factor in stratified analyses or meta-regressions. Estimates for some comparisons were imprecisely estimated as noted by the wide CrIs, specifically bleeding and hepatic events. This was due to very low numbers (i.e., 0 or 1 ) of subjects reported as experiencing such events in one or more treatment arms in the included trials. Therefore, findings for these comparisons need to be interpreted with caution.

Furthermore, our analyses included only one study (the ALFA-0701 trial) that evaluated GO in the recommended dose and schedule (i.e., $3 \mathrm{mg} / \mathrm{m}^{2}$ fractionated dose schedule). As such, our results may have understated the true efficacy of GO, impacting the estimates derived from the comparisons. Data on health-related quality of life or patient-reported outcomes were sparsely reported in the included trials. Because of this, a comparative assessment of the impact of GO + DA and other induction therapies on these outcomes was not feasible in our analyses. Health-related quality of life outcomes are increasingly being included as end points in clinical trials, especially in oncology. Thus, future NMAs may be able to incorporate and analyze these outcomes.

There were limited instances of comparisons with both direct and indirect evidence. As a result, it was not possible to assess the validity of the consistency assumption (i.e., that the direct and indirect evidence are equivalent 
and in agreement). In the few outcomes with direct and indirect evidence for some comparisons (relapse, bleeding and hepatic effects, and infection), the estimates of treatment effects from the direct and indirect evidence were in agreement in all instances.

Lastly, the literature search that informed the analyses was conducted in September 2015. An updated literature search conducted in January 2018 yielded only one new RCT; a Phase II study of idarubicin and cytarabine with clofarabine or fludarabine in patients with newly diagnosed AML [37]. If the analysis were updated to include this study, it is likely it would have had minimal impact on the conclusions.

\section{Conclusion}

Findings from this SLR and NMA indicate that GO + DA is an effective combination therapy compared with other agents used as induction therapy in the management of newly diagnosed de novo or secondary AML. In the base-case analyses, GO + DA was associated with significantly greater OS and RFS compared with most evaluated treatments, but a higher rate of relapse compared with GO + FLAG-I. Base-case safety analyses showed higher rates of bleeding and hepatic events with GO + DA versus comparator treatments, consistent with GO's known safety profile. There were no differences seen for the other efficacy or safety outcomes. These findings are consistent with what is known about the efficacy and safety profile of GO. Results of the two subgroup analyses were generally consistent with the base-case.

\section{Future perspective}

The treatment landscape for newly diagnosed AML has only begun to change in the past year with the approval of GO and other new agents. These approvals provide new, viable treatment options for this hard-to-treat disease. However, recent advancements in cancer therapy, such as the development of targeted agents, will further expand the treatment options available, not only for the general AML population but also for subgroups of patients with certain characteristics (such as unfavorable cytogenetics or presence of co-morbid conditions) that may modulate response to currently available therapy. It will be important to assess the comparative efficacy and safety of these new agents as more data on their use become available.

Supplementary data

To view the supplementary data that accompany this paper please visit the journal website at: www.futuremedicine.com/doi/full/ 10.2217/fon-2018-0512

Financial \& competing interests disclosure

This study was sponsored by Pfizer Inc. K Fahrbach is an employee of Evidera Inc., which provides consulting and other research services to pharmaceutical, medical device and related organizations. In his salaried position, he works with a variety of companies and organizations, and is precluded from receiving payment or honoraria directly from these organizations for services rendered. AO Ashaye, Y Xu and Z Khankhel were employees of Evidera Inc. at the time of this study. Evidera was paid by Pfizer Inc. to conduct this study and develop this manuscript. ME Orme works for ICERA Consulting Ltd., which received funding from Pfizer Inc. for this work. R Mokgokong, K Lang, JC Cappelleri and C Mamolo are employees of Pfizer Inc., and hold stock/stock options in Pfizer Inc. The authors have no other relevant affiliations or financial involvement with any organization or entity with a financial interest in or financial conflict with the subject matter or materials discussed in the manuscript apart from those disclosed.

No writing assistance was utilized in the production of this manuscript.

Open access

This work is licensed under the Attribution-NonCommercial-NoDerivatives 4.0 Unported License. To view a copy of this license, visit http://creativecommons.org/licenses/by-nc-nd/4.0/ 


\section{Background}

- There have been few advances, and thus limited options of induction therapy for the management of newly diagnosed acute myeloid leukemia (AML). Few head-to-head trials have compared the efficacy and safety of gemtuzumab ozogamicin (GO) + daunorubicin-cytarabine (DA) to other induction therapies in this patient population.

\section{Methods}

- A systematic literature review was undertaken based on published guidelines. Searches were conducted in Embase, MEDLINE, the Cochrane Central Register of Controlled Trials and the Database of Abstracts of Reviews of Effects to identify randomized controlled trials published between 1 January 2000 and 29 September 2015.

- Estimates of relative treatment effects were derived from a Bayesian network meta-analysis framework for efficacy outcomes (overall survival [OS], relapse-free survival [RFS], induction death, complete remission, relapse, resistant disease) and safety outcomes (infection, bleeding events, skin effects and hepatic events).

- Base-case analyses included all studies providing evidence for a specific outcome of interest. Subgroup analyses included: an analysis limited to studies evaluating a $100 \%$ de novo AML population; an analysis limited to studies evaluating GO-based therapies (i.e., in combination with DA or any other therapy) to provide a GO versus no GO comparison.

Results

- In the base-case analyses, GO + DA was associated with significantly greater OS and RFS compared with most comparator treatments, but a higher rate of relapse compared with one comparator. Base-case safety analyses showed higher rates of bleeding and hepatic events with GO + DA versus comparator treatments. There were no differences seen for the other efficacy or safety outcomes. These findings are consistent with what is known about the efficacy and safety profile of GO.

- Results of the two subgroup analyses were generally consistent with the base-case analyses. Similar significant OS and RFS benefits were seen with GO + DA compared with evaluated therapies in patients with de novo AML. In GO versus no-GO analyses, OS and RFS were significantly higher and relapse rate was significantly lower with the GO-based regimen. There was no difference with regard to complete remission, and results for resistant disease were equivocal.

- Findings from this systematic literature review and network meta-analysis indicate that GO + DA provides additional significant OS and RFS benefit compared with evaluated comparator therapies used in the management of newly diagnosed (de novo or secondary) AML.

\section{References}

Papers of special note have been highlighted as: • of interest; $\bullet \bullet$ of considerable interest

1. American Cancer Society. Key statistics for acute myeloid leukemia (2018). www.cancer.org/cancer/acute-myeloid-leukemia/about/key-statistics.html

2. Wander SA, Levis MJ, Fathi AT. The evolving role of FLT3 inhibitors in acute myeloid leukemia: quizartinib and beyond. Ther. Adv. Hematol. 5(3), 65-77 (2014).

3. Döhner H, Estey E, Grimwade D et al. Diagnosis and management of AML in adults: 2017 ELN recommendations from an international expert panel. Blood 129(4), 424-447 (2017).

4. Yates JW, Wallace HJ Jr, Ellison RR, Holland JF. Cytosine arabinoside (NSC-63878) and daunorubicin (NSC-83142) therapy in acute nonlymphocytic leukemia. Cancer Chemother. Rep. 57(4), 485-488 (1973).

5. Lichtman MA. A historical perspective on the development of the cytarabine (7 days) and daunorubicin (3 days) treatment regimen for acute myelogenous leukemia: 2013 the 40th anniversary of 7 + 3. Blood Cells Mol. Dis. 50(2), 119-130 (2013).

- An informative review of the use and history of the $7+3$ regimen, that for 40 years has been the backbone of high intensity induction therapy in newly diagnosed acute myelogenous leukemia (AML).

6. Dillman RO, Davis RB, Green MR et al. A comparative study of two different doses of cytarabine for acute myeloid leukemia: a Phase III trial of Cancer and Leukemia Group B. Blood 78(10), 2520-2526 (1991).

7. Schiffer CA, Dodge R, Larson RA. Long-term follow-up of cancer and leukemia Group B studies in acute myeloid leukemia. Cancer 80(11 Suppl.), 2210-2214 (1997).

8. Röllig C, Müller-Tidow C, Hüttmann A et al. Sorafenib versus placebo in addition to standard therapy in younger patients with newly diagnosed acute myeloid leukemia: Results from 267 patients treated in the randomized placebo-controlled SAL-Soraml trial. Blood 124(21), 6 (2014).

9. Burnett A, Russell N, Hills $\mathrm{R}$ et al. A randomised comparison of the novel nucleoside analogue sapacitabine with low-dose cytarabine in older patients with acute myeloid leukaemia. Leukemia 29(6), 1312-1319 (2015). 
10. Dinndorf PA, Andrews RG, Benjamin D, Ridgway D, Wolff L, Bernstein ID. Expression of normal myeloid-associated antigens by acute leukemia cells. Blood 67(4), 1048-1053 (1986).

11. US FDA. NDA 21-174: Mylotarg (gemtuzumab ozogamicin) Injection. Drug Approval Package. Company: Wyeth-Ayerst. Approval Date: 5/17/2000. New Drug Application No.: 21-174 (2000). www.accessdata.fda.gov/drugsatfda_docs/nda/2000/21174_Mylotorg.cfm

12. US FDA. Proposed text of labeling for the drug - annotated: Mylotarg ${ }^{\top M}$ (gemtuzumab ozogamicin for Injection) (2000). www.accessdata.fda.gov/drugsatfda_docs/label/2000/21174lbl.pdf

13. Hills RK, Castaigne S, Appelbaum FR et al. Addition of gemtuzumab ozogamicin to induction chemotherapy in adult patients with acute myeloid leukaemia: a meta-analysis of individual patient data from randomised controlled trials. Lancet Oncol. 15(9), 986-996 (2014).

-. This gemtuzumab ozogamicin (GO) meta-analysis supported the positive results reported in the AML-15, AML-16 and ALFA-0701 trials (improvements in 2-year estimated event-free survival [EFS], relapse-free survival [RFS] and overall survival $[O S]$ with the addition of GO vs daunorubicin-cytarabine [DA] alone).

14. US FDA. FDA approves Mylotarg for treatment of acute myeloid leukemia (2017). www.fda.gov/NewsEvents/Newsroom/PressAnnouncements/ucm574507.htm

15. Higgins JPT, Green S. (Eds). Cochrane Handbook for Systematic Reviews of Interventions. Version 5.1.0[updated March 2011]. The Cochrane Collaboration (2011). www.handbook.cochrane.org

16. Hutton B, Salanti G, Caldwell DM et al. The PRISMA extension statement for reporting of systematic reviews incorporating network meta-analyses of health care interventions: checklist and explanations PRISMA extension for network meta-analysis. Ann. Intern. Med. 162(11), 777-784 (2015).

17. Higgins JP, Altman DG, Gotzsche PC et al. The Cochrane Collaboration's tool for assessing risk of bias in randomised trials. BMJ 343 , d5928 (2011).

18. Caldwell DM, Ades AE, Higgins JP. Simultaneous comparison of multiple treatments: combining direct and indirect evidence. $B M J$ 331(7521), 897-900 (2005).

19. Dias S, Sutton AJ, Ades A, Welton NJ. Evidence synthesis for decision making 2: a generalized linear modeling framework for pairwise and network meta-analysis of randomized controlled trials. Med. Decis. Making 33(5), 607-617 (2013).

\section{-• A key methodology paper on network meta-analysis as a tool for evidence synthesis in medicine.}

20. Dias S, Welton NJ, Sutton AJ, Ades AE. Evidence synthesis for decision making 1: introduction. Med. Decis. Making 33(5), 597-606 (2013).

21. Lu G, Ades AE. Combination of direct and indirect evidence in mixed treatment comparisons. Stat. Med. 23(20), 3105-3124 (2004).

22. National Institute for Health and Care Excellence (NICE). Guide to the Methods of Technology Appraisal 2013 (2013). www.nice.org.uk/process/pmg9/resources/guide-to-the-methods-of-technology-appraisal-2013-pdf-2007975843781

23. Salanti G, Ades AE, Ioannidis JP. Graphical methods and numerical summaries for presenting results from multiple-treatment meta-analysis: an overview and tutorial. J. Clin. Epidemiol. 64(2), 163-171 (2011).

24. Hoyle MW, Henley W. Improved curve fits to summary survival data: application to economic evaluation of health technologies. $B M C$ Med. Res. Methodol. 11, 139 (2011).

25. Parmar MK, Torri V, Stewart L. Extracting summary statistics to perform meta-analyses of the published literature for survival endpoints. Stat. Med. 17(24), 2815-2834 (1998).

26. Lunn D, Spiegelhalter D, Thomas A, Best N. The BUGS project: evolution, critique and future directions. Stat. Med. 28(25), 3049-3067 (2009).

27. Brooks SPG. General methods for monitoring convergence of iterative simulations. J. Comput. Graph. Stat. 7(4), 434-455 (1998).

28. Gelman A. Inference and monitoring convergence. In: Markov Chain Monte Carlo in Practice. Gilks WR, Richardson S, Spiegelhalter DJ (Eds). Chapman and Hall, London, 131-143 (1996).

29. Spiegelhalter DJ, Best NG, Carlin BP, van der Linde A. Bayesian measures of model complexity and fit. J. R. Stat. Soc. Series B 64(4), 583-639 (2002).

30. Oberg G, Killander A, Bjoreman M et al. Long-term follow-up of patients $>$ or $=60 \mathrm{yr}$ old with acute myeloid leukaemia treated with intensive chemotherapy. Eur. J. Haematol. 68(6), 376-381 (2002).

31. Ohtake S, Miyawaki S, Fujita $\mathrm{H}$ et al. Randomized study of induction therapy comparing standard-dose idarubicin with high-dose daunorubicin in adult patients with previously untreated acute myeloid leukemia: the JALSGAML201 study. Blood 117(8), 2358-2365 (2011).

32. Rowe JM, Neuberg D, Friedenberg W et al. A Phase 3 study of three induction regimens and of priming with GM-CSF in older adults with acute myeloid leukemia: a trial by the Eastern Cooperative Oncology Group. Blood 103(2), 479-485 (2004).

33. Pautas C, Merabet F, Thomas X et al. Randomized study of intensified anthracycline doses for induction and recombinant interleukin-2 for maintenance in patients with acute myeloid leukemia age 50 to 70 years: results of the ALFA-9801 study. J. Clin. Oncol. 28(5), 808-814 (2010). 
34. Castaigne S, Pautas C, Terre C et al. Effect of gemtuzumab ozogamicin on survival of adult patients with de-novo acute myeloid leukaemia (ALFA-0701): a randomised, open-label, Phase 3 study. Lancet 379(9825), 1508-1516 (2012).

-. Reported the results of the key ALFA-0701 trial that led to the approval of GO in de novo AML.

35. Büchner T, Schlenk RF, Schaich M et al. Acute myeloid leukemia (AML): different treatment strategies versus a common standard arm-combined prospective analysis by the German AML Intergroup. J. Clin. Oncol. 30(29), 3604-3610 (2012).

- A large study with an interesting design: it introduced a common treatment arm $(7+3$ regimen) as a benchmark for comparison, with patients randomized from five different trials.

36. Kharfan-Dabaja MA, Hamadani M, Reljic T et al. Gemtuzumab ozogamicin for treatment of newly diagnosed acute myeloid leukaemia: a systematic review and meta-analysis. Br. J. Haematol. 163(3), 315-325 (2013).

- This early meta-analysis reported that the addition of GO to conventional chemotherapy improved RFS and EFS; these findings were corroborated the following year by Hills et al.'s meta-analysis.

37. Jabbour E, Short NJ, Ravandi F et al. A randomized Phase 2 study of idarubicin and cytarabine with clofarabine or fludarabine in patients with newly diagnosed acute myeloid leukemia. Cancer 123(22), 4430-4439 (2017).

38. Anderson JE, Kopecky KJ, Willman CL et al. Outcome after induction chemotherapy for older patients with acute myeloid leukemia is not improved with mitoxantrone and etoposide compared to cytarabine and daunorubicin: a Southwest Oncology Group study. Blood 100(12), 3869-3876 (2002).

39. Burnett AK, Milligan D, Goldstone A et al. The impact of dose escalation and resistance modulation in older patients with acute myeloid leukaemia and high risk myelodysplastic syndrome: the results of the LRF AML14 trial. Br. J. Haematol. 145(3), 318-332 (2009)

40. Burnett AK, Hills RK, Milligan D et al. Identification of patients with acute myeloblastic leukemia who benefit from the addition of gemtuzumab ozogamicin: results of the MRC AML15 trial. J. Clin. Oncol. 29(4), 369-377 (2011).

41. Burnett AK, Russell NH, Hills RK et al. Addition of gemtuzumab ozogamicin to induction chemotherapy improves survival in older patients with acute myeloid leukemia. J. Clin. Oncol. 30(32), 3924-3931 (2012).

42. Castaigne $S$, Chevret $S$, Archimbaud E et al. Randomized comparison of double induction and timed-sequential induction to a "3+7" induction in adults with AML: long-term analysis of the Acute Leukemia French Association (ALFA) 9000 study. Blood 104(8), 2467-2474 (2004).

43. Goldstone AH, Burnett AK, Wheatley K, Smith AG, Michael Hutchinson R, Clark RE. Attempts to improve treatment outcomes in acute myeloid leukemia (AML) in older patients: the results of the United Kingdom Medical Research Council AML11 trial. Blood 98(5), 1302-1311 (2001).

44. Holowiecki J, Grosicki S, Robak T et al. Addition of cladribine to daunorubicin and cytarabine increases complete remission rate after a single course of induction treatment in acute myeloid leukemia. Multicenter, Phase III study. Leukemia 18(5), 989-997 (2004).

45. Holowiecki J, Grosicki S, Giebel S et al. Cladribine, but not fludarabine, added to daunorubicin and cytarabine during induction prolongs survival of patients with acute myeloid leukemia: a multicenter, randomized Phase III study. J. Clin. Oncol. 30(20), 2441-2448 (2012).

46. Juliusson G, Höglund M, Karlsson $\mathrm{K}$ et al. Increased remissions from one course for intermediate-dose cytosine arabinoside and idarubicin in elderly acute myeloid leukaemia when combined with cladribine. A randomized population-based Phase II study. $B r$. J. Haematol. 123(5), 810-818 (2003).

47. Mandelli F, Vignetti M, Suciu S et al. Daunorubicin versus mitoxantrone versus idarubicin as induction and consolidation chemotherapy for adults with acute myeloid leukemia: the EORTC and GIMEMA groups study AML-10. J. Clin. Oncol. 27(32), 5397-5403 (2009).

48. Ossenkoppele GJ, Stussi G, Maertens J et al. Addition of bevacizumab to chemotherapy in acute myeloid leukemia at older age: a randomized Phase 2 trial of the Dutch-Belgian Cooperative Trial Group for Hemato-Oncology (HOVON) and the Swiss Group for Clinical Cancer Research (SAKK). Blood 120(24), 4706-4711 (2012). 
